\title{
О СТАРТУЮЩЕЙ ПЛАВУЧЕЙ ТУРБУЛЕНТНОЙ СТРУЕ
}

\author{
(Представил И. Эпик)
}

Рассматривается течение, реализующееся в свободновосходящих под действием силы тяжести конвективных потоках в атмосфере, а также в некоторых технологических процессах (при напылении полупроводников и т. д.). Для течений султанообразного типа в иностранной литературе используется термин «plume», а для фазы начального развития «starting plume». Такое движение впервые изучалось Б. Я. Зельдовичем $\left[{ }^{1}\right]$, определившим предельные законы для стационарных свободновосходящих турбулентных потоков. Согласно экспериментальным данным, в некотором диапазоне чисел Рейнольдса в этом случае устанавливается течение, имеющее вид всплывающего вихревого кольца над

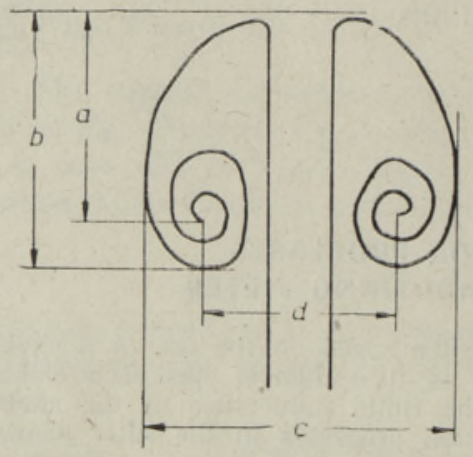

Рис. 1. Форма стартующей плавучей струи.

свободновосходящей струей (рис. 1). Зафиксирована автомодельность развития всплывающего вихревого кольца (подобного термику) с равномерно возрастающей во времени плавучестью $\left[{ }^{2}\right]$, что позволяет использовать для описания стартующей плавучей турбулентной струи (СПТС) расширяющуюся систему координат $\left[{ }^{3}\right]$.

В качестве исходной системы уравнений выберем уравнения движения и переноса тепла для несжимаемой жидкости. в приближении Буссинеска. Замыкание уравнений осуществим введением турбулентных аналогов коэффициентов кинематической вязкости $v^{*}$ и температуропроводности $a^{*}$, для которых примем

$$
\begin{aligned}
& v^{*}=v_{t}(x, r) L(t) V(t), \\
& a^{*}=a_{t}(x, r) L(t) V(t) .
\end{aligned}
$$

Здесь $L(t)$ - характерный размер элемента, $V(t)$ - его характерная скорость, $v_{t}, a_{t}$ - функции координат, формы которых определяются сравнением результатов расчета и эксперимента. С учетом сделанных предположений запишем исходные уравнения задачи при $v_{t}, a_{t}=$ const:

$$
\begin{gathered}
\frac{\partial \zeta}{\partial t}+\frac{\partial}{\partial r}(v \zeta)+\frac{\partial}{\partial x}(u \zeta)+\beta g \frac{\partial T}{\partial r}=v^{*}(t)\left[\frac{\partial^{2} \zeta}{\partial x^{2}}+\frac{\partial^{2} \zeta}{\partial r^{2}}+q \frac{\partial}{\partial r}\left(\frac{\zeta}{r}\right)\right], \\
\zeta=-\frac{1}{r^{q}}\left[\frac{\partial^{2} \psi}{\partial x^{2}}+\frac{\partial^{2} \psi}{\partial r^{2}}-\frac{q}{r} \frac{\partial \psi}{\partial r}\right],
\end{gathered}
$$

где $\zeta-$ вихрь, $\psi-$ функция тока, $v=-r^{-q} \partial \psi / \partial x, u=r^{-q} \partial \psi / \partial r-$ 
компоненты скорости, $\dot{\beta}$ - коэффициент теплового расширения, $g$ ускорение свободного падеиия, $x, r-$ оси цилиндрической системы координат в осесимметричной задаче $(q=1)$ или оси декартовой системы координат в плоской задаче $(q=0), t$ - время.

Исходное уравнение притока тепла выпишем в. виде

$$
\begin{gathered}
\frac{\partial T}{\partial t}+v \frac{\partial T}{\partial r}+u \frac{\partial T}{\partial x}=\frac{v^{*}(t)}{\operatorname{Pr}_{t}}\left(\frac{\partial^{2} T}{\partial x^{2}}+\frac{\partial^{2} T}{\partial r^{2}}+\frac{q}{r} \frac{\partial T}{\partial r}\right)+ \\
+\left[\frac{\partial}{\partial x}\left(u_{0} T_{0}\right)+\frac{\partial}{\partial r}\left(r^{q} v_{0} T_{0}\right)\right] .
\end{gathered}
$$

Здесь $T$ - температура, $\operatorname{Pr}_{t}=v^{*} / a^{*}$, а последнее слагаемое в скобках определяет приток тепла из следа, регулирующий «подпитку» области всплывающего вихревого кольца в процессе его формирования. След СПТС описывается аналитическими решениями $\left(u_{0}, v_{0}, T_{0}\right)$ в приближении пограничного слоя для стационарных свободновосходящих струй. Им посвящены работы $\left[{ }^{4-7}\right]$. Далее мы непосредственно используем результаты $\left[{ }^{6,7}\right]$, соответствующие осесимметричному и плоскому случаям.

Интегрируя (4) по объему $V(T \rightarrow 0$ при $x \rightarrow \infty)$, получим

$$
(2 \pi)^{q} \beta g \iint_{s} r^{q} T d r d x=Q_{1} t+Q_{0}, \quad\left\{s^{*}: 0<r<\infty, x^{*}<x<\infty\right\},
$$

$$
Q_{1}=(2 \pi)^{q} \beta g \int_{0}^{\infty} r^{q} u_{0} T_{0} d r=\text { const. }
$$

Далее положим $Q_{0}=0$.

Значение $x^{*}$ задает нижнюю границу области, занимаемой автомодельно развивающейся частью СПТС.

Преобразуем уравнения (2)-(4)

$$
\begin{gathered}
x_{1}=x / L(t), \quad r_{1}=r / L(t), \quad u_{1}=u / V(t), \quad v_{1}=v / V(t), \\
\zeta_{1}=\zeta L(t) / V(t), \quad \psi_{1}=\psi / L^{q+1} V(t), \quad T_{1}=T_{\beta g} / \theta(t), \quad V(t)=d L / d t
\end{gathered}
$$

и приведем их с учетом (1) к безразмерному виду:

$$
\begin{gathered}
\frac{\partial \zeta_{1}}{\partial s}-p \zeta_{1}+\left(u_{1}-x_{1}\right) \frac{\partial \zeta_{1}}{\partial x_{1}}+\left(v_{1}-r_{1}\right) \frac{\partial \zeta_{1}}{\partial r_{1}}-\frac{q v_{1} \zeta_{1}}{r_{1}}= \\
=v_{t}\left[\frac{\partial^{2} \zeta_{1}}{\partial x_{1}^{2}}+\frac{\partial^{2} \zeta_{1}}{\partial r_{1}^{2}}+q \frac{\partial}{\partial r_{1}}\left(\frac{\zeta_{1}}{r_{1}}\right)\right]-\frac{\partial T_{1}}{\partial r_{1}} \\
\frac{\partial^{2} \psi_{1}}{\partial x_{1}^{2}}+\frac{\partial^{2} \psi_{1}}{\partial r_{1}^{2}}-\frac{q}{r_{1}} \frac{\partial \psi_{1}}{\partial r_{1}}=-r_{1}^{q} \zeta_{1}, \\
\frac{\partial T_{1}}{\partial s}-F T_{1}+\left(u_{1}-x_{1}\right) \frac{\partial T_{1}}{\partial x_{1}}+\left(v_{1}-r_{1}\right) \frac{\partial T_{1}}{\partial r_{1}}= \\
=\frac{v_{t}}{P r_{t}^{\prime}}\left[\frac{\partial^{2} T_{1}}{\partial x_{1}^{2}}+\frac{\partial^{2} T_{1}}{\partial r_{1}^{2}}-\frac{q}{r_{1}} \frac{\partial T_{1}}{\partial r_{1}}\right]+ \\
+\left[\frac{\partial}{\partial x_{1}}\left(u_{0} T_{0}\right)+\frac{1}{r_{1}^{q}} \frac{\partial}{\partial r_{1}}\left(r_{1}^{q} v_{0} T_{0}\right)\right] \mathrm{e}^{-3(13 / 9)^{q} s}, \quad s=\ln \left(t / t_{0}\right) / p
\end{gathered}
$$

В автомодельном режиме зависимости масштабов от времени имеют вид $L \sim t^{\alpha}, \Theta \sim t^{\beta}$. Значения постоянных сведены в табл. 1. Запишем граничные условия и условия нормировки 


\begin{tabular}{c|c|c|c|c}
\hline$q$ & $p$ & $F$ & $\alpha_{*}$ & $\beta_{*}$ \\
\hline 0 & 1 & 1 & 1 & -1 \\
1 & $4 / 3$ & $5 / 3$ & $3 / 4$ & $-5 / 4$
\end{tabular}

\begin{tabular}{c|c|c|c|c|c}
\hline $\begin{array}{c}\text { Харак- } \\
\text { тери- } \\
\text { стики }\end{array}$ & \multicolumn{4}{|c|}{ Данные расчета при $v_{t}$} & $\begin{array}{c}\text { Данные } \\
\text { экспе- } \\
\text { римен- } \\
\text { та }\left[{ }^{2}\right]\end{array}$ \\
\hline \multicolumn{1}{|c|}{0,03} & 0,04 & 0,05 & 0,06 & \\
$b / c$ & 0,75 & 0,90 & 0,86 & 0,83 & 0,90 \\
$d / c$ & 0,64 & 0,63 & 0,62 & 0,58 & 0,79 \\
$a / c$ & 0,37 & 0,46 & 0,43 & 0,39 & 0,56
\end{tabular}

$$
\begin{aligned}
& \psi_{1}=\zeta_{1}=\frac{\partial T_{1}}{\partial r_{1}}=0 \quad\left(r_{1}=0\right), \\
& \psi_{1}=\zeta_{1}=T_{1}=0
\end{aligned}
$$

$\left(x_{1}, r_{1} \in S_{1}^{*}\right.$, где $S_{1}^{*}-$ граница объема $\left.V_{1}\right)$,

$$
(2 \pi)^{q} \iint_{S_{1}^{*}} r_{1}^{q} T_{1} d x_{1} d r_{1}=Q_{1}
$$

Автомодельный режим в расчете может быть достигнут при отсутствии зависимости от безразмерного времени. Из (9) видно, что при больших значения $s$ приток тепла из следа становится исчезающе малым, и автомодельный режим развития СПТС будет описываться решением краевой задачи (7)-(11). Численное решение задачи проводилось по явной двухслойной схеме с аппроксимацией конвективных членов по Аракаве методом установления. Для решения уравнения Пуассона (8) использовался метод «последовательной перерелаксации» $\left[{ }^{8}\right]$. Структура схемы подробно описана в $\left.{ }^{9}\right]$. Начиная с некоторого момента времени, когда теплосодержание достигало значения $Q_{1}$, применялась мультипликативная поправка для $T_{1}$, получаемая для каждого временного слоя из условия (11). Расчеты проводились на квадратной сетке с числом ячеек $42 \times 31$ для $v_{t}=0,03 ; 0,04 ; 0,05 ; 0,06$ и $\operatorname{Pr}_{t}^{\prime}=0,5 ; 1$ и 2. При этом принималось $Q_{1}=1, x^{*}=1$.

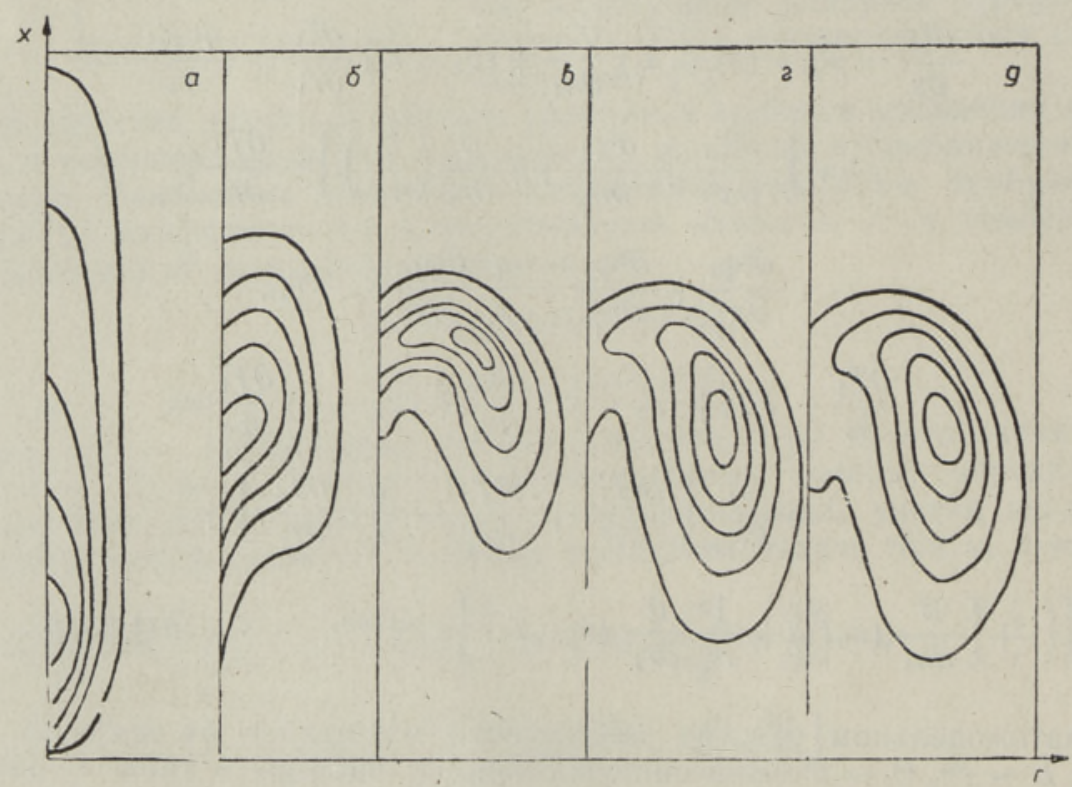

Рис. 2. Изолинии в разные моменты времени $\left(10,30,50,70\right.$ и $90 \%$ от $\left.T_{m}\right)$ при $s=30(a), 60(б), 90(в), 120($ (ट) и $150(\partial)$. 
Сравнение рассчитанной структуры всплывающего вихревого кольца (рис. 2) с наблюдаемой СПТС $\left[{ }^{2}\right]$ указывает на их удовлетворительное качественное совпадение. В расчетах зафиксированы грибовидная и тороидальная формы распределения температуры. Как и в случае термика, максимум температуры смещается с уменьшением коэффициента турбулентной вязкости $v$ (с увеличением $\operatorname{Pr}_{t}^{\prime}$ ) дальше от центра симметрии, в результате чего грибовидная форма переходит в тороидальную. В табл. 2 приведены расчетные (при $\operatorname{Pr}^{\prime}=1$ ) и экспериментальные значения геометрических характеристик СПТС. Сравнение этих данных говорит о совпадении общей формы поля температуры при $v_{t}=0,04$ (характеристика $b / c$ ). Некоторое отличие в поведении максимума температуры (характеристики $d / c$ и $a / c$ ) связано, по-видимому, с наличием схемной вязкости, влекущей более плавное по сравнению с реальным изменение температуры в области больших градиентов на границе температурного поля.

\section{Л И Т Е Р А Т У РА}

1. Зельдович Б. Я. Ж. эксперим. и теор. физ., 7, № 12, 1463 (1937).

2. Shilen, $D$. J. Phys. Fluids, 19, № 8, $1089-1098$ (1976).

3. Lilly D. K. J. Atmos. Sci., 23, 83-98 (1964).

4 Васильченко И. В. Тр. Гл. геофиз. обсерв., вып. 93, 29-35 (1959).

5. Гостинцев Ю. А., Суханов Л. А., Солодовник А. Ф. В кн.: Турбулентные струйные течения. Таллин, «Валгус», 1982, с. $74-79$.

6. Chia Shun Yih, Feng Wu. Phys. Fluids, 24, № 5, 794-801 (1981).

7. Капланский $\Phi$. Б. В кн.: Турбулентные струйные течения. Таллин, «Валгус», 1982, c. $80-86$.

8. Численные методы в динамике жидкостей (под ред. Г. Вагрц и Ж. Смолдерен). М., «Мир», 1981.

9. Капланский Ф. Б. В кн.: Процессы рассеивания вредных примесей в приземном слое атмосферы. Таллин, «Валгус», 1976, с. 124-140.

\section{Институт термофизики и электрофизики Академии наук Эстонской ССР}

Поступила в редакцию 29 июня 1982

\section{F. KAPLANSKI}

\section{KONVEKTIIVSE TURBULENTSE JOA LEVIKUST}

On koostatud ja arvutusmeetodil lahendatud isemodelleeruv konvektiivse turbulentse joa leviku ülesanne. Teoreetilisi tulemusi on võrreldud katseandmetega, mille pōhjal on määratud turbulentse viskoossuse mooduli efektiivne väärtus - 0,04.

\section{F. KAPLANSKI}

\section{ON TURBULENT STARTING PLUME}

A two-dimensional problem of turbulent starting plume is considered on the assumption that the cap of starting plume can be treated as a thermal with buoyancy constant increase. Experimental researches of plumes carried by Shilen D. J. proved this assumption. The equations of vorticity and heat transfer for a turbulent motion of incompressible fluid are transformed into a non-dimensional form which includes the requirements to reach the similarity regime. The system of equations is solved numerically for various values for spatially constant eddy viscosity and heat diffusion coefficients by taking the exact solution for wake at the rear of the plume. The obtained similarity distributions of temperature for a cap of starting plume are compared with available experimental data $\left(\operatorname{Re} \approx 10^{2}\right)$. The results of computations show the existence of a corresponding value of the non-dimensional eddy vișcosity coefficient 0,04 , 